\title{
EFFECT OF SINGLE AND COMBINED INOCULATIONS OF POTATO PLANTS WITH FOUR FUSARIUM SPECIES ON WILT SEVERITY, PLANT GROWTH, AND PRODUCTION
}

\author{
a,bBoutheina Mejdoub-Trabelsi*, bHayfa Jabnoun-Khiareddine, ${ }^{b}$ Rania Aydi Ben Abdallah, bNawaim Ammar, \\ bMejda Daami-Remadi \\ a Higher School of Agriculture of Kef, University of Jendouba, Tunisia. \\ b UR13AGR09, Production Horticole Intégrée au Centre-Est Tunisien, Centre Régional des Recherches en Horticulture et \\ Agriculture Biologique de Chott-Mariem, Université de Sousse, 4042,Chott-Mariem, Tunisia.
}

\section{ART I C L E I N F O}

\section{Article history}

Received: March 21, 2020

Revised: May 25, 2020

Accepted: August 05, 2020

\section{Keywords}

Aggressiveness

Competition

Cultivar response

Fusarium wilt

Mixed inoculations

Potato

Synergy

\section{A B S T R A C T}

Potato (Solanum tuberosum L.) Fusarium wilt is an increasingly serious disease in many potato-growing regions worldwide and notably in Tunisia. Fusarium sambucinum, F. oxysporum, F. solani, and F. graminearum are the main causal agents with $F$. oxysporum being the most aggressive on plants. Co-infection with this Fusarium species complex is frequently observed. Cultivar responses to different pathogen mixtures can give additional information on the relative aggressiveness of the different complexes. This study may lead to a better understanding of how interactions between the four Fusarium species may affect disease incidence and severity. Interactions between these Fusarium species was evaluated using single and combined inoculations (15 different Fusraium inocula) onto potato the two cultivars Spunta and Oceania. The tested inocula were evaluated for their effects on leaf yellowing and necrosis, vascular discoloration severity, potato growth and production. Necrosis severity noted 75 days postplanting induced by three different inocula C2-1 (F. sambucinum $+F$. solani $),$ C2-2 (F. solani $+F$. oxysporum), and C3-3 (F. sambuinum $+F$. oxysporum $+F$. graminearum $)$ were found to be the highly aggressive inoculation treatments. Overall, all mixed inocula including $F$. sambucinum showed increased aggressiveness levels. The two cultivars exhibited differential response to the different tested Fusarium mixtures and behaved in the majority as sensitive to moderately sensitive to C1-1, C1- 4, C2$1, \mathrm{C} 2-2, \mathrm{C} 2-3, \mathrm{C} 2-4, \mathrm{C} 2-5, \mathrm{C} 2-6, \mathrm{C} 3-1, \mathrm{C} 3-3, \mathrm{C} 3-4$ and $\mathrm{C} 4$ treatments. This study clearly demonstrated that Fusarium wilt of potato is expected to be more severe when more than one Fusarium species is present. The relative predominance of $F$. sambucinum may reflect its competitive potential in mixture and its significant involvement in potato Fusarium wilt severity.

Corresponding Author: Boutheina Mejdoub-Trabelsi

Email: boutheinam2002@yahoo.fr

(C) The Author(s) 2020.

\section{INTRODUCTION}

Potato (Solanum tuberosum L.) losses attributed to fungal diseases are becoming the major worrying constraints faced by potato growers worldwide. Among the widely encountered biotic problems, Fusarium wilt is an increasingly serious soilborne disease as the causal agents (various Fusarium species) are difficult to control due to their soilborne origin and inevitable occurrence (Munkvold, 2016).

Many potato organs (tubers, roots, stems, etc) may be infected by Fusarium species leading to root and tuber rots and eventual plant wilt witling in addition to tuber 
contamination by mycotoxins (Pernezny et al., 2014). These pathogens are responsible for severe vascular wilts, root and crown rots in a diverse group of crop plants (Enya et al., 2007). In fact, Fusarium wilt diseases rank among the most devastating diseases, constituting a significant agricultural threat (Fisher et al., 2012). This problem persists throughout the world and is prevalent in the most potato-growing areas leading to $30-50 \%$ yield losses and decreased quality and safety of tubers intended for consumption (Ommati and Sharifi, 2008; Kerkeni et al., 2013). Fusarium wilt has been identified as one of the most yield limiting disease of potato, worldwide (Ommati et al., 2013).

Fusarium oysporum is infamous for causing vascular wilt diseases in over 100 different crops (Edel-Hermann and Lecomte, 2019). In Tunisia, F. oxysporum and F. solani are the main species involved in plant wilting (Daami-Remadi et al., 2006; Ayed et al., 2006). In addition, they are frequently associated to tuber dry rot (Daami-Remadi and El Mahjoub, 2004). Infection of plants may be initiated on roots through natural openings or wounds before invading the xylem vascular tissues. This infection process leads to plant stunting, reduced growth, leaf yellowing, vascular browning, and eventual total wilting of severely infected stems (Ayed et al., 2006).

The literature on this disease is not extensive, although its occurrence in many potato producing countries (Saremi and Amiri, 2010; Xue et al., 2013). In addition, Fusarium Wilt is most often associated with infections with other bio-agressors such as the root lesion nematode and other fungal and bacterial agents responsible for the known early potato dying syndrome (Daami-Remadi et al., 2008). In fact, when multiple pathogens are present, interactions may result in additive or synergistic increases in disease incidence and severity as well as decreases in yield (Hou et al., 2020). In this context, previous studies demonstrated that Verticillium dahliae and Pectobacterium spp. can individually have significant detrimental effects on potato production, but when they are present together, their destructive potential can be significantly increased (Rowe, 1987). Another study reported that plants co-inoculated with both $V$. dahliae and $P$. carotovorum subsp. carotovorum exhibited more severe chlorosis and stem soft rot symptoms as compared to plants inoculated with either pathogen alone (Rahimian, 1984). In the same way, Dung et al. (2013) indicated that co-infection by Pectobacterium and $V$. dahliae can result in increased potato early dying severity.
For an efficient Fusarium wilt control, the use of resistant varieties is the most reliable approach especially in terms of preservation and respect of environment and human health. Esfahani (2005) recorded a varied response of potato varieties to Fusarium wilt caused by F. sulphureum, F. solani and F. oysporum. In Tunisia, many research efforts attempted to select a potato variety resistant to Fusarium species involved in this disease but no combined resistance was found but only some varieties displayed tolerance to two Fusarium species at most (Daami-Remadi et al., 2006; Mejdoub-Trabelsi et al., 2012). In addition, Selection pressures attributed to the host, as well as inter- and intra-specific competition drive the evolution of fungal genomes (Rampersad, 2020).

Faced with this situation, the study of the interaction occurring between all Fusarium species complex is required. Obtaining a high level of resistance to the whole pathogen complex seems to be closely related to the relationship characterizing the involved Fusarium species. Their frequently observed coexistence in nature encourages us to associate them through mixed inoculations, thus imitating the concrete situation encountered under natural conditions during potato growing cycle. Our previous research (MejdoubTrabelsi and Ben Abdallah, 2016), more focused on Fusarium dry rot disease, has led to some very interesting and promising results where we demonstrated that disease development and severity is more severe when more than one Fusarium species is present suggesting an additive effect between them during their interaction ( $F$. oxysorum, $F$. solani, $F$. sambucinum, and F. graminearum). However, to the best of our knowledge, the relationships between pathogen species responsible for Fusarium wilt disease have not been previously explored.

Therefore, the major aims of current investigation were to (i) elucidate the type of interaction occurring between the four Fusarium species causing potato Fusarium wilt and to assess their effects on wilt development and severity based on single and mixed inoculations and (ii) determine the relative aggressiveness of the tested inocula depending on potato cultivars.

\section{MATERIALS AND METHODS \\ Fungal inoculum}

Plant infection was performed using a multiple set of inoculum, i.e., a single Fusarium species, a mixture of 
two, three and four Fusarium species. Spore suspensions were prepared by culturing Fusarium spp. in Potato Dextrose Broth (PDB). After incubation for 7 days at 25 ${ }^{\circ} \mathrm{C}$ under continuous shaking at $150 \mathrm{rpm}$, the cultures were filtered through two layers of cheesecloth to remove mycelium and then through two layers of Whatman No. 1 filter paper. The obtained suspension was filtered and equal volumes of conidial suspensions from each Fusarium species were mixed and the final concentration of the combined inoculum was adjusted to $10^{7} \mathrm{CFU} / \mathrm{mL}$ using a Malassez hemacytometer. The different types of inocula used for plant inoculation were detailed in Table 1.

Table 1. Single and mixed inocula of Fusarium spp. used for inoculation of potato plants.

\begin{aligned} & \hline \multicolumn{1}{c}{ Type of inoculum } Involved Fusarium species \\ & \hline Monospecific inoculum Fusarium solani \\ & C $1-1-2$ F. sambucinum \\ & C1-3 F. graminearum \\ & C1-4 F. oxysporum \\ & Bispecific inoculum $2-1$ F. solani + F. sambucinum \\ & C2-2 F. solani + F. oxysporum \\ & C2-3 F. solani + F. graminearum \\ & C2-4 F. oxysporum + F. sambucinum \\ & C2-5 F. oxysporum + F. graminearum \\ & C2-6 F. graminearum + F. sambucinum \\ & C3-1 F. solani + F. oxysporum + F. sambucinum \\ & C3-2 F. solani + F. oxysporum + F. graminearum \\ & C3-3 F. oxysporum + F. sambucinum + F. graminearum \\ & C3-4 F. solani + F. graminearum + F. sambucinum \\ & C4 4 F. solani + F. sambucinum + F. graminearum + F. oxysporum \\ & \hline\end{aligned}

\section{Plant material}

Potato cvs. Spunta and Oceania tubers, known to be susceptible to Fusarium wilt (Ayed et al., 2006), were used in this pot experiment. They were previously stored at $6{ }^{\circ} \mathrm{C}$ for two months before being used. They were superficially disinfected using a sodium hypochlorite solution diluted at $10 \%$ during $5 \mathrm{~min}$, rinsed with tap water and air dried. They were maintained under ambient conditions for germination.

\section{Plant inoculation}

The mixed Fusarium species were evaluated on potato plants for their relative aggressiveness as Fusarium wilt agents compared to single inoculations. Seed tubers, showing optimal germination, were planted in individual pots containing a mixture of perlite and peat $(1: 3 \mathrm{v} / \mathrm{v})$. Two weeks after emergence, potato seedlings were infected with the different Fusarium spp. inocula (applied singly or in combination) by watering each pot with $100 \mathrm{ml}$ of a conidial suspension $\left(10^{7} \mathrm{CFU} / \mathrm{mL}\right)$. Uninoculated control plants were watered with $100 \mathrm{ml}$ of sterile distilled water only (Daami-Remadi and El Mahjoub, 2004). All treated pots were placed under greenhouse conditions $\left(18-25^{\circ} \mathrm{C}, 14 \mathrm{~h}\right.$ light) for 60 days and watered regularly enough to avoid drought stress. Ten plants were used per individual treatment. The whole experiment was repeated twice.

Fusarium wilt severity was assessed, 60 days postinoculation, based on the intensity of leaf yellowing and necrosis. This parameter representing the intensity of foliar symptoms was noted using the following arbitrary 0-4 scale (Kerkeni et al., 2013) where $0=$ asymptomatic leaves, $1=$ Wilted leaves, 2 = Leaves showing unilateral yellowing, 3 = Leaves showing unilateral necrosis, and 4 $=$ Dead leaves (Figure 1).

The second disease severity parameter is the index of the vascular discoloration estimated through 
longitudinal sections of stems and measurement of the length of this staining from the collar to the end of the stalk. This evaluation was performed per stem and the average for all stems was calculated per plant. Then, this parameter was classified according to an arbitrary scoring scale of the vascular discoloration (browning) index ranging from 0 to 4 (Figure 2) as follows where 0 : No vascular browning; 1: Browning $\leq 1 \mathrm{~cm} ; 2: 1 \mathrm{~cm}<$ Vascular browning $\leq 3 \mathrm{~cm} ; 3: 3 \mathrm{~cm}<$ Vascular browning $\leq 5 \mathrm{~cm}$; : Vascular browning $>5 \mathrm{~cm}$.
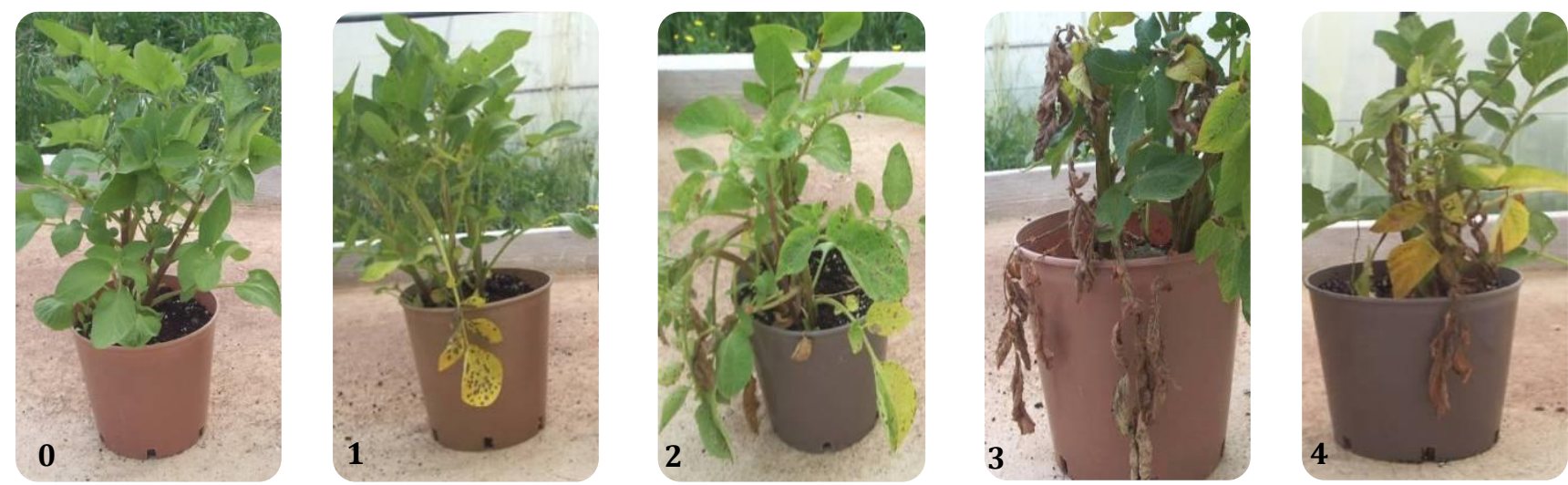

Figure 1. Arbitrary scale used for the evaluation of Fusarium wilt severity on potato plants.
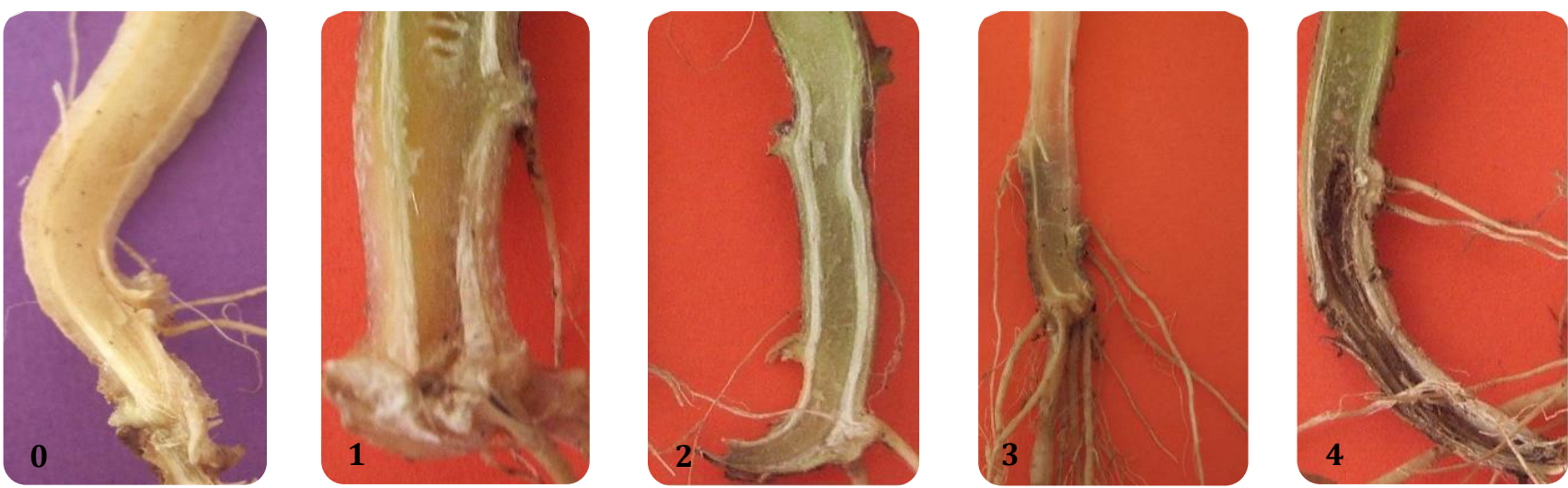

Figure 2. Rating scale used for the evaluation of the vascular browning severity on longitudinally sectioned potato stems.

Other growth parameters such as the fresh weight of the aerial part and tubers were noted to evaluate the effect of simple and mixed inoculations on plant growth and production.

The classification of fungal treatments (single and mixed inocula) tested on plants, as Fusarium wilt agents, was performed based on necrosis index records using the following arbitrary scale:

$\checkmark$ Highly aggressive (HA): Necrosis index $\geq 2$

$\checkmark$ Moderately aggressive (MA): $1<$ Necrosis index $<2$

$\checkmark$ Non aggressive (NA): Necrosis index $\leq 1$

The classification of potato cultivars for their response to the tested simple and mixed infections, was carried out based on necrosis index records using the following arbitrary scale where: $\checkmark$ Sensitive: (S): Necrosis index $\geq 2$

$\checkmark$ Moderately sensitive (MS): $1<$ Necrosis index $<2$

$\checkmark$ Tolerant (T): Necrosis index $\leq 1$

\section{Statistical analyses}

A factorial analysis of variance (ANOVA) was performed to determine the significance of the main factors and their interactions using a completely randomized factorial design with two tested factors i.e. potato cultivars and fungal treatments (tubers inoculated with single or mixed inocula and the uninoculated control). Mean separations were performed using the Duncan's multiple range test (at $P<0.05$ ) (Mejdoub-Trabelsi et al., 2012). 


\section{RESULTS}

All plants inoculated with Fusarium spp. showed typical symptoms of Fusarium wilt. Thus, all fungal treatments, tested singly or in mixtures, were found to be pathogenic on potato plants. However, wilt severity varied depending on parameters noted, nature of the inoculum used for plant inoculation and cultivars tested.

\section{Leaf necrosis severity}

Leaf necrosis severity, noted 75 days post-planting, varied significantly depending on tested cultivars and fungal treatments used for plant inoculation.

As for the relative effects of tested inoculation treatments on this parameter, Figure 3 shows that on cv. Spunta, 11 treatments out of the 15 tested induced the development of necrosis signs of significantly similar severity where the registered necrosis indexes varied between 1.8 and 2.5 . These records concerned the mono-specific treatment C14, 06 bi-specific inocula (C2-4, C2-5, C2-2, C2-1, C2-6 and C2-3), 03 tri-specific treatments (C3-3, C3-4 and C3-1) and the tetra-specific inculum (C4). The treatments C1-1, C1-2 C1-3, and C3-2 induced the least severe necrosis signs.

As for $\mathrm{cv}$. Oceania response to the different inoculation treatments, the recorded necrosis severity was less than that noted on cv. Spunta and did not exceed 2.5. Only plants infected with $\mathrm{C} 3-3$ and C4 inocula showed a significantly higher necrosis index than the other treatments. All bi-specific inocula, except C2-3, induced a significantly comparable necrosis severity. However, the tri-specific inocula C3-1 and C3-2, the mono-specific ones including C1-1, C1-2 and C1-3 and bispecific treatment $\mathrm{C} 2-3$, induced the lowest necrosis indexes.

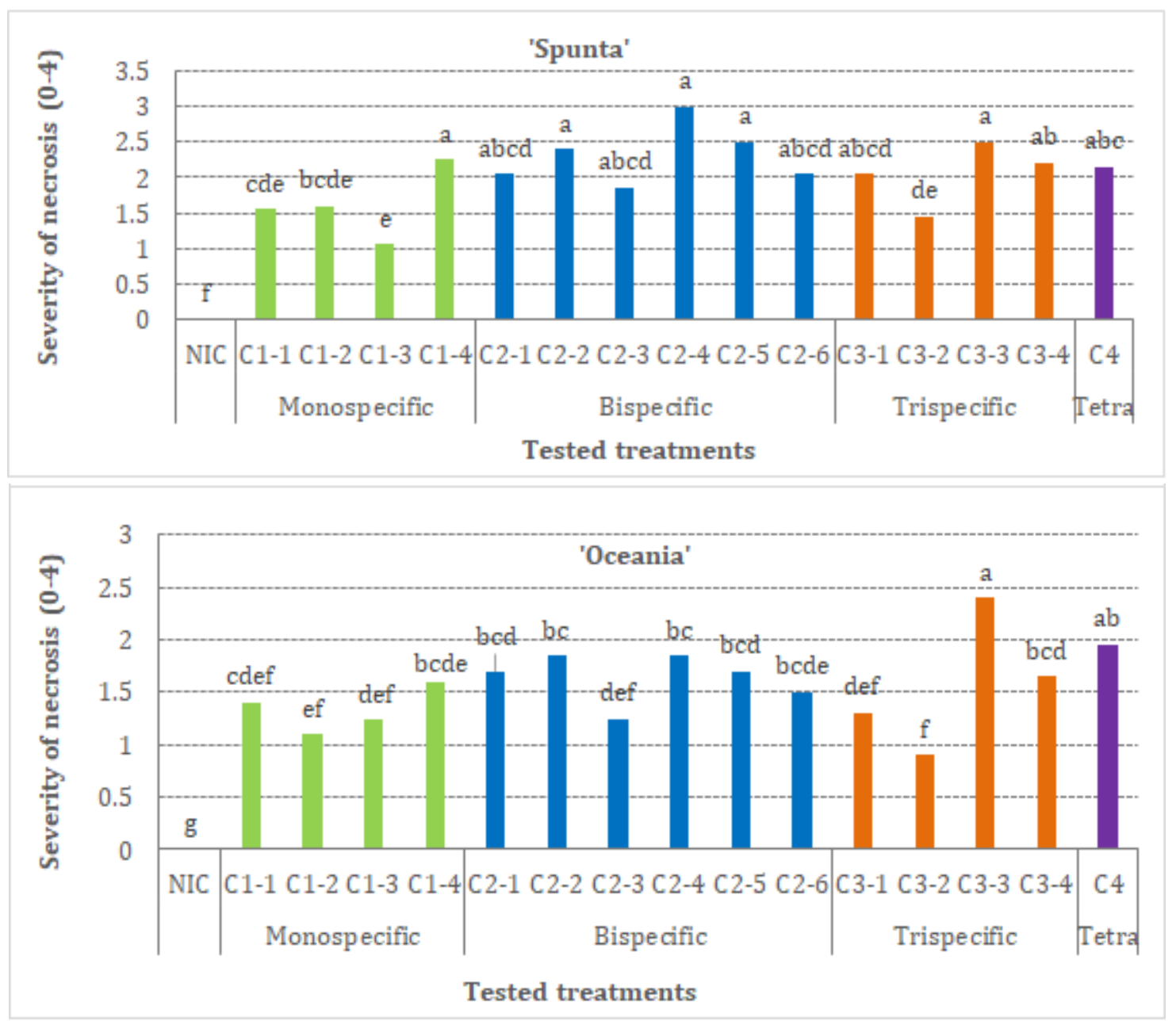

Figure 3. Effect of single and mixed inoculations by four Fusarium species on leaf necrosis severity noted on two potato cultivars 75 days post-planting.

Bars sharing the same letter are not significantly different according to Duncan's Multiple Range Test at $P \leq 0.05$. NIC: Uninoculated control, Tetra: Tetraspecific, The composition of all tested inoculation treatments is detailed in Table 1. 


\section{Vascular browning severity}

Fusarium wilt severity, evaluated based on the index of vascular browning, varied significantly depending on tested fungal treatments. Data given in Figure 4 showed that for all cv. Spunta plants, the vascular browning index did not exceed 2 where only 05 treatments out of the 15 tested led to the development of a vascular browning with an index ranging between 1.55 and 2 . These treatments are composed of the tetra-specific inoculum (C4), 02 tri-specific treatments (C3-3, C3-4), the bi-specific inoculum $\mathrm{C} 2-4$ and the mono-specific inoculum C1-4.

It is important to note that plants inoculated with C3-
2 Fusarium species complex showed the lowest browning index (0.5) which was significantly comparable to the uninoculated control and which was 4 times lower than that noted on those infected with C1-4. As for cv. Oceania response to the different inoculation treatments, 05 out of the 15 tested inocula induced a vascular browning with an index ranging between 1.2 and 2 . The concerned treatments were C2-5, C3-3, C4, C1-4 and C2-4. However, two trispecific (C3-1 and C3-2), four bi-specific (C2-2, C2-3, C2-6 and C2-1) and three mono-specific (C1-2, C1-3 and C1-1) inocula induced a vascular browning with a comparable severity the uninoculated control.

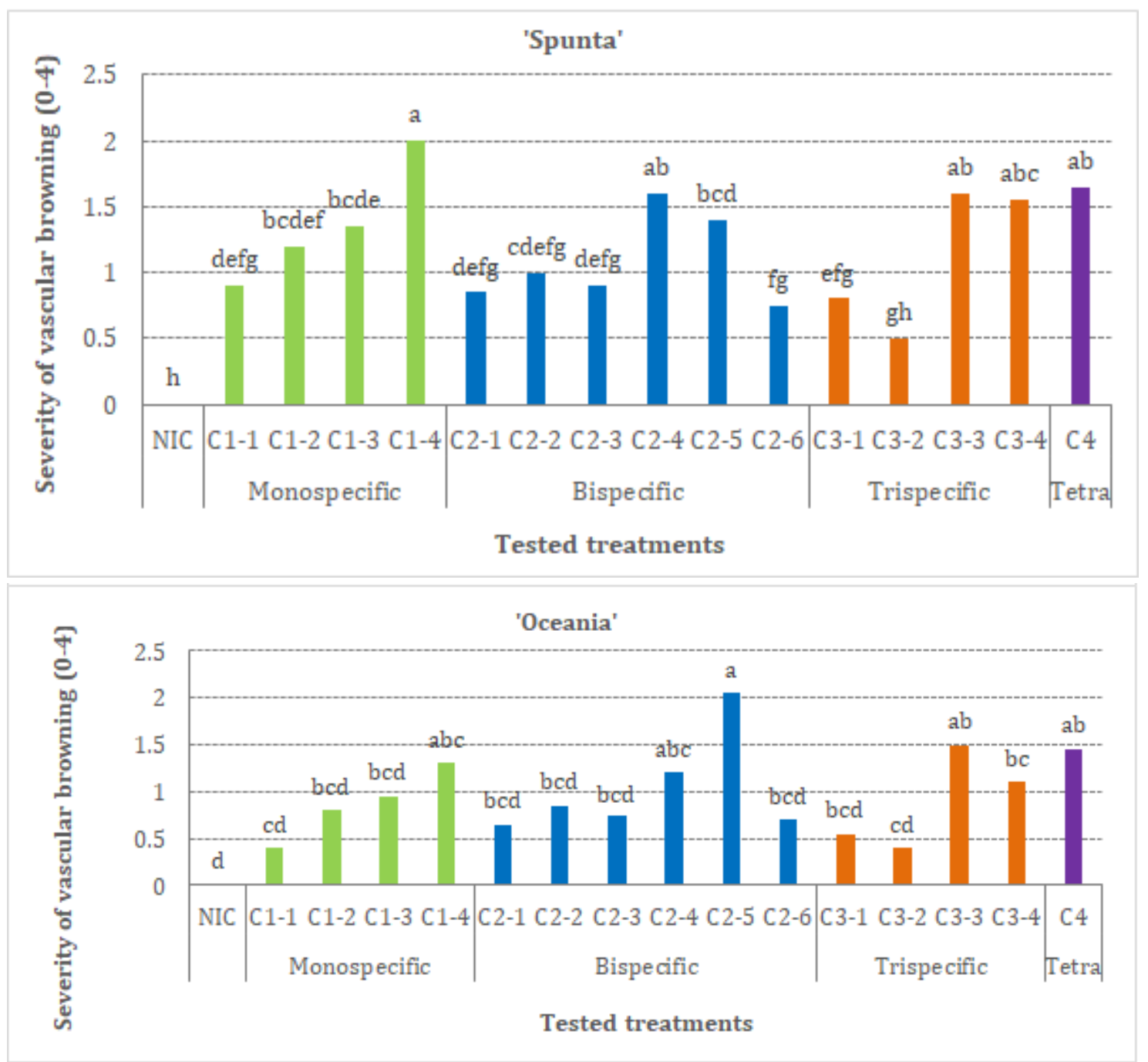

Figure 4. Effect of single and mixed inoculations by four species of Fusarium on the vascular browning severity noted on two potato cultivars 75 days post-planting.

Bars sharing the same letter are not significantly different according to Duncan's Multiple Range Test at $P \leq 0.05$.

NIC: Uninoculated control, Tetra: Tetraspecific, The composition of all tested inoculation treatments is detailed in Table 1. 
As the leaf necrosis was the sole disease severity parameter which varied at the same time depending on cultivars used and tested inoculation treatments, all inoculum types (mono, bi, tri and tetra-specific) were ranked based on their relative levels of aggressiveness on the tested cultivars (Table 2).

As for ratings of single-specific inocula as wilting agents on cv. Spunta, only C1-4 (F. oxysporum) out of the four mono-specific inocula tested was found to be highly aggressive. However, on cv. Oceania, all mono-specific inocula were moderately aggressive except C1-2 (F. sambucinum), which was not-aggressive on potato plants. As for the ranking of of the bi-specific treatments when challenged to $\mathrm{cv}$. Spunta, the six inocula were found to be highly aggressive whereas on cv. Oceania, they were in majority moderately aggressive except $\mathrm{C} 2-1 \quad(F$. sambucinum $+F$. solani) and C2-2 (F. solani $+F$. oxysporum) which were shown to be highly aggressive. As for wilt severity induced by the tri-specific inocula, C3-3 (F. sambucinum + F. oxysporum + F. graminearum) was ranked as highly aggressive on both varieties (Table 2, Figure. 5).

Table 2. Classification of mono, bi, tri and tetra-specific Fusarium inocula based on their aggressiveness on two potato cultivars based on leaf necrosis severity noted 75 days post-planting.

\begin{tabular}{|c|c|c|}
\hline Cultivar/ Type of inoculum & \multicolumn{2}{|c|}{ cv. Spunta/cv. Oceania } \\
\hline \multicolumn{3}{|l|}{ monospecific Inoculum } \\
\hline C1-1: F. sol & MA & MA \\
\hline C1-2: F. sam & MA & NA \\
\hline C1-3: F.gra & NA & MA \\
\hline C1-4: F. oxy & $\mathrm{HA}$ & MA \\
\hline \multicolumn{3}{|l|}{ Bispecific Inoculum } \\
\hline C2-1: F. sam+F. sol & HA & HA \\
\hline C2-2: F. sol+F. oxy & HA & HA \\
\hline C2-3: F. sol+F. gra & HA & MA \\
\hline C2-4:F.sam+F. oxy & HA & MA \\
\hline C2-5: F. oxy+F.gra & HA & MA \\
\hline C2-6: F. sam+F.gra & HA & MA \\
\hline \multicolumn{3}{|l|}{ Trispecific Inoculum } \\
\hline C3-1: F. sam+F. sol+F. oxy & HA & MA \\
\hline C3-2: F. sol+F. oxy+F. gra & MA & NA \\
\hline C3-3: F. sam+F. oxy+F. gra & HA & HA \\
\hline C3-4: F. sam+F. sol+F. gra & HA & MA \\
\hline \multicolumn{3}{|l|}{ Tetraspecific Inoculum } \\
\hline C4: F. sam $+F \cdot s o l+F . o x y+F . g r a$ & HA & MA \\
\hline
\end{tabular}

HA: Highly aggressive: Necrosis index $\geq 2$; MA: Moderately aggressive: $1<$ Necrosis index < 2; NA: Not aggressive: Necrosis index $\leq 1$; F. sol: F. Solani ; F. gra: F. graminearum; F. sam: F. sambuinum; F. oxy: F. oxysporum
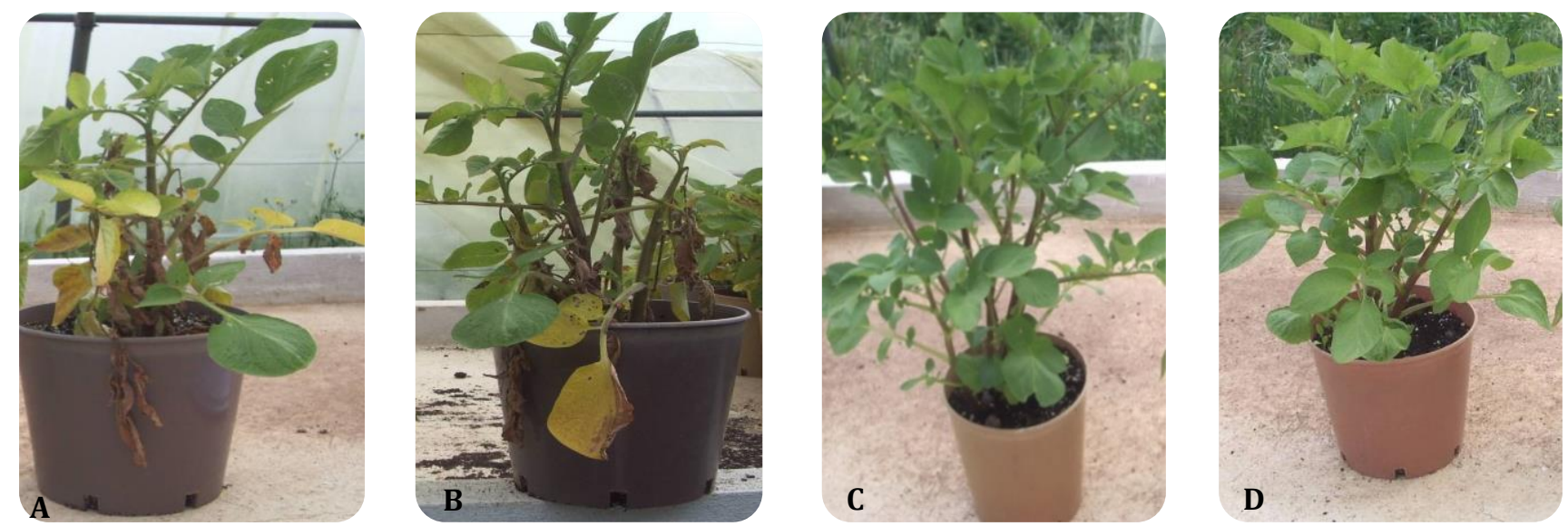

Figure 5. Fusarium wilt severity caused by the tri-specific inoculum composed of Fusarium sambucinum, $F$. oxysporum and F. graminearum behaving as highly aggressive on cv. Spunta (A) and cv. Oceania (B) as compared to the uninoculated controls ( $C$ and $\mathrm{D}$, respectively). 
In addition, two tri-specific treatments among the four tested, namely C 3-1 (F. sambucinum + F. solani $+F$. oxysporum) and C3-4 (F. sambucinum $+F$. solani $+F$. graminearum), were found to be moderately aggressive on cv. Oceania. Interestingly, the tri-specific C3-2 treatment $(F$. oxysporum $+F$. solani $+F$. graminearum $)$ was ranked as non-aggressive on cv. Oceania and as moderately aggressive on cv. Spunta.

Effect of simple or mixed inoculations with Fusarium spp. on potato growth and production parameters

Analysis of variance performed for the three studied parameters (weight of the aerial part, roots and tubers) revealed a highly significant interaction (at $P<0.01$ ) between the two tested factors (cultivars and fungal treatments).

\section{Aerial part fresh weight}

The effect of mono and multi-specific inoculations with Fusarium species based on the fresh weight of the stems is shown in Figure 6. This figure shows that all single and mixed inocula tested, regardless of the varieties used, had significantly reduced the fresh weight of the aerial part by 27.51 to $59.26 \%$ compared with the uninoculated control.

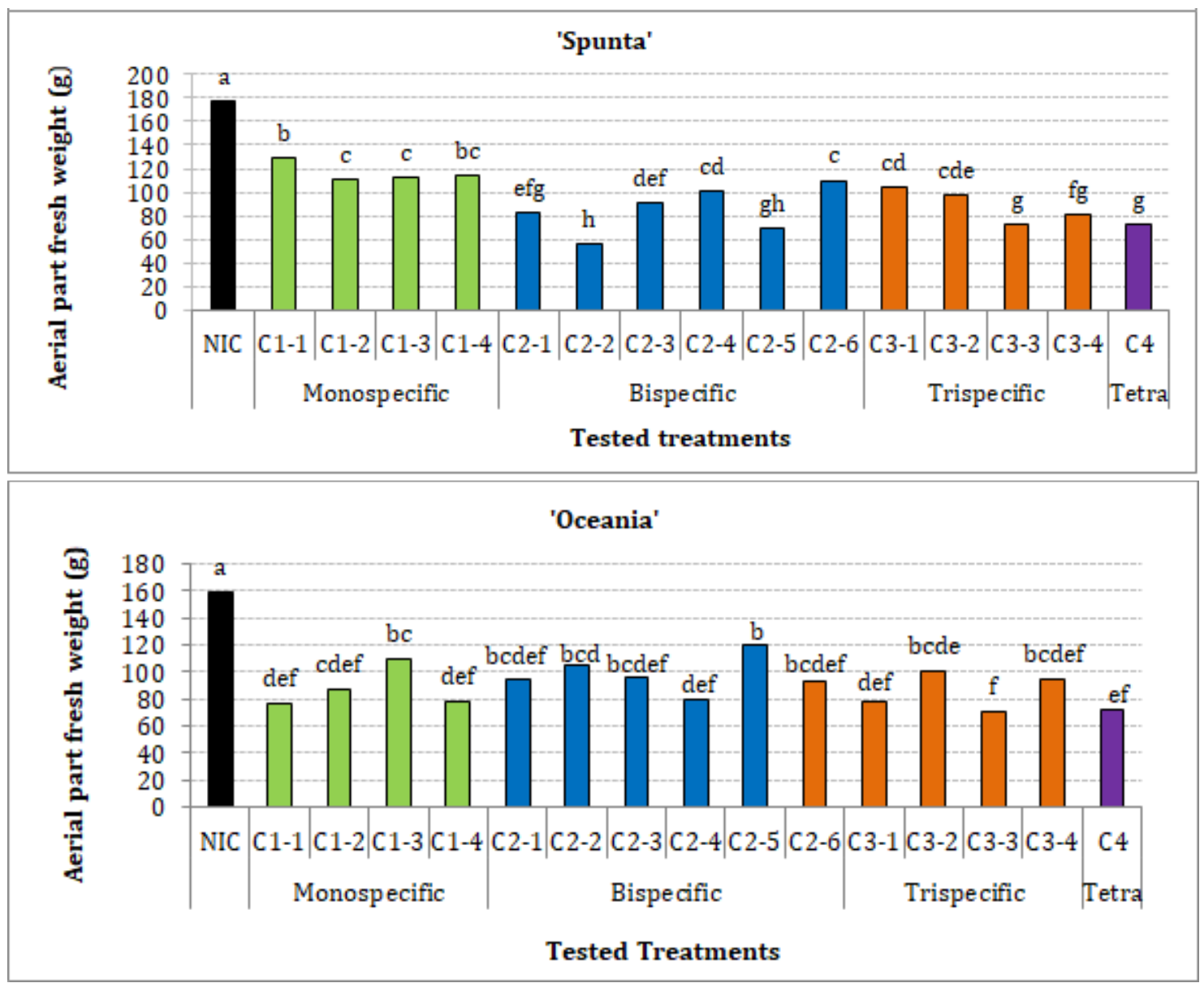

Figure 6. Effect of single and mixed inoculations by four Fusarium species on the aerial part fresh weight noted on two potato cultivars 75 days post-planting

Bars sharing the same letter are not significantly different according to Duncan's Multiple Range Test at $P \leq 0.05$.

NIC: Uninoculated control, Tetra: Tetraspecific, The composition of all tested inoculation treatments is detailed in Table 1.

As for the specific effects of the tested mono and multispecific inocula based on the fresh weight of the aerial part, the inoculation of cv. Spunta with the single-specific inocula had reduced this parameter by 13.49 to $41.25 \%$ compared to control. However, on cv. Oceania, monospecific inoculations led 3.69 to $47.55 \%$ lesser aerial weight relative to control. As for the bi-specific inocula challenged to cv. Spunta, the treatment C2-2 (F. solani + F. oxysporum) reduced the weight of the aerial part of $72.81 \%$ versus control. 
The tri-specific inocula tested on cv. Spunta decreased this parameter by 36.92 to $61.81 \%$ compared to control plants whereas reduction ranged between 36.25 and $62.57 \%$ when challenged to $\mathrm{cv}$. Oceania. Inoculation by the tetraspecific inoculum led to $60 \%$ decrease in Spunta versus 59.91\% in Oceania.

It is important to note that regardless of cultivars used and inoculations performed, mixed inocula C2-2 ( $F$. solani $+F$. oxysporum $)$, C3-3 ( $F$. sambucinum $+F$. oxysporum $+F$. graminearum) and $C 4$ induced the highest decreases in the aerial part weight estimated at $54.90,52.26$ and $57.51 \%$, respectively. These three inocula were followed by four bi-specific fungal treatments including C2-1 (F. sambucinum + F.solani), C2-3 (F. solani+ F. graminearum), C2-4 (F. sambucinum + $F$. oxysporum), and C2-5 (F. oxysporum $+F$. graminearum) leading to 47.55 to $49.77 \%$ decrease in this parameter as compared to control. These four inocula were followed by another group of four treatments including C1-2 (F. sambucinum) and three tri-specific treatments namely C3-1 (F. sambucinum $+F$. solani + F oxysporum $),$ C3-2 (F. solani $+F$. oxysporum $+F$. graminearum), and C3-4 (F. sambucinum + F. solani $+F$. graminearum) where the recorded reductions ranged between 42.92 and $44.20 \%$. The three mono-specific inocula C1-3 (F. graminearum), C1-4 (F. oxysporum) and C1-1 (F. solani) limited this growth parameter by 27.51 to $33.66 \%$ relative to control.

\section{Root fresh weight}

The root fresh weight varied significantly (at $P<0.05$ ) depending on cultivars used and fungal treatments tested. The respective effects of the different inocula on tested cultivars are presented in Figure 7.

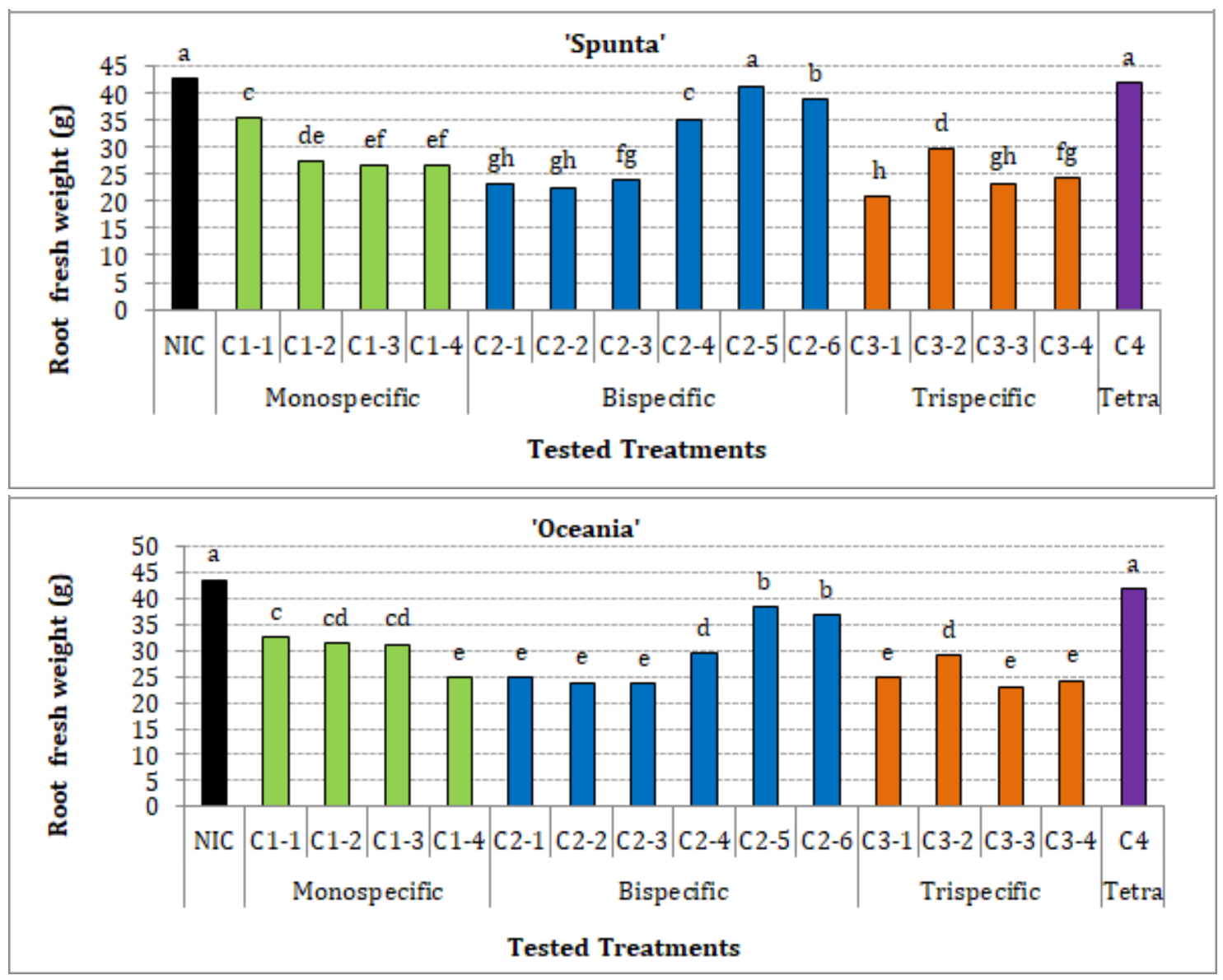

Figure 7. Effect of single and mixed inoculations by four Fusarium species on the root fresh weight noted on two potato cultivars 75 days post-planting

Bars sharing the same letter are not significantly different according to Duncan's Multiple Range Test at $P \leq 0.05$.

NIC: Uninoculated control, Tetra: Tetraspecific , The composition of all tested inoculation treatments is detailed in Table 1. 
Figure 7 shows that the monospecific inocula challenged to $\mathrm{cv}$. Spunta led to 22.97 to $51.20 \%$ reduced root fresh weight versus control as compared to 26.67 to $49.37 \%$ noted on cv. Oceania. As for the effects of the bi-specific inoculation treatments, the drop of the roots fresh weight varied between 5.19 and $51.01 \%$ in cv. Spunta and from 9.45 to $50.18 \%$ in cv. Oceania. However, plant inoculations with the tri-specific treatments showed 25.33-52.87 and 29.85-49.12\% reduced root fresh weight in cvs. Spunta and Oceania, respectively, as compared to their respective controls. In contrast, fresh root weight decreases attributed to the tetra-specific infection varied from 1.87 to $4.74 \%$ on Spunta and from 0.81 to $14.14 \%$ on Oceania.

It is important to note that, regardless of cultivars used, 03 out of the 06 bi-specific inocula tested namely C2-1 (F. solani + F. sambucinum), C2-2 (F. solani + F. oxysporum $)$, and C2-3 (F. solani + F. graminearum), 03 out of 04 trispecific treatments namely C3-1 (F. sambucinum $+F$. solani $+F$. oxysporum), C3-3 (F.sambucinum $+F$. oxysporum + F. graminearum), and C3-4 (F. sambucinum + $F$. solani + F.graminearum) induced the highest decreases in the root fresh weight estimated at $43.41-46.96 \%$ less than control.

These fungal treatments were followed by two monospecific inocula namely C1-3 (F. graminearum) and C1-4 (F. oxysporum) which lowered this parameter by 38.33 and $37.41 \%$, respectively. The third group of relatively important inocula, based on the fresh root weight reduction compared to control, is composed of C12 (F. sambucinum) and C3-2 (F. solani + F. oxysporum + F. graminearum) with 32.05 and $30.37 \%$, respectively.

These fungal treatments were followed by $\mathrm{C} 1-1$ (F. solani) and C2-4 (F. sambucinum $+F$. oxysporum) leading to 23.19 and $25.54 \%$ decreases in this growth parameter, respectively. Treatments C2-5 (F. oxysporum $+F$. graminearum), C2-6 (F. sambucinum + F. graminearum) and $\mathrm{C} 4$ reduced this parameter by 5.59 to $11.18 \%$ versus control.

\section{Tuber fresh weight}

This parameter varied significantly depending on cultivars tested and fungal treatments used for plant inoculation; a highly significant interaction (at $P \leq 0.01$ ) was recorded between these two factors.
Concerning the relative effects of single and multispecific inocula on this production parameter, Figure 8 shows that all tested treatments led to significant decreases in tuber yields in both cultivars as compared to control. In fact, following inoculation of cv. Spunta with the tested monospecific inocula, there was a decrease in the tuber fresh weight estimated at 2.36 to $50.42 \%$ depending on Fusarium species. However, for cv. Oceania, the recorded yield reductions ranged from 21.36 to $36.22 \%$.

As for the effects of bi-specific inocula, Figure 8 shows that the tuber weight loss varied between 0.73 and $20.38 \%$ on cv. Spunta and between 24.34 and $50.41 \%$ on cv. Oceania. Tri-specific inocula applied to both cultivars led to reduced tuber weight ranging from 0.21 to $17.32 \%$ on cv. Spunta and from 18.98 to $30.27 \%$ on cv. Oceania. The tuber yield decrease on cvs. Spunta and Oceania attributed to the tetra-specific infection was about 4 and $7 \%$, respectively. It should be noted that, regardless of potato cultivars used, inoculations with the treatment C1-4 (F. oxysporum) led to $47.74 \%$ decrease in this production parameter as compared to the uninoculated control. Infections with C1-3 (F. graminearum), C2-1 (F. sambucinum + F. solani), and C31 (F. sambucinum + F. solani + F. oxysporum) inocula had reduced this parameter by 36.66 to $41.31 \%$. Six inocula followed this group in terms of tuber weight reduction i.e. C1-1 (F. solani), C1-2 (F. sambucinum), C2-2 (F. solani + F. oxysporum), C2-3 (F. solani + F. graminearum), C2-4 (F. sambucinum $+F$. oxysporum), and the tetra-specific inoculum $\mathrm{C} 4$ where the recorded decreases ranged between 31.42 and $34.51 \%$. As for the specific effects of the remaining treatments, the recorded drops in the tubers fresh weight, as compared to control, ranged between 24 and $28 \%$.

\section{Cultivar response to single and mixed infections with Fusarium species}

Table 3 shows that both tested cultivars behaved in the majority as sensitive to moderately sensitive to C1-1, C14, C2-1, C2-2, C2-3, C2-4, C2-5, C2-6, C3-1, C3-3, C3-4 and $\mathrm{C} 4$ infections. The only cases of tolerance were recorded on cv. Spunta inoculated with C1-3 and cv. Oceania challenged with C1-2 and C3-2 inocula. 


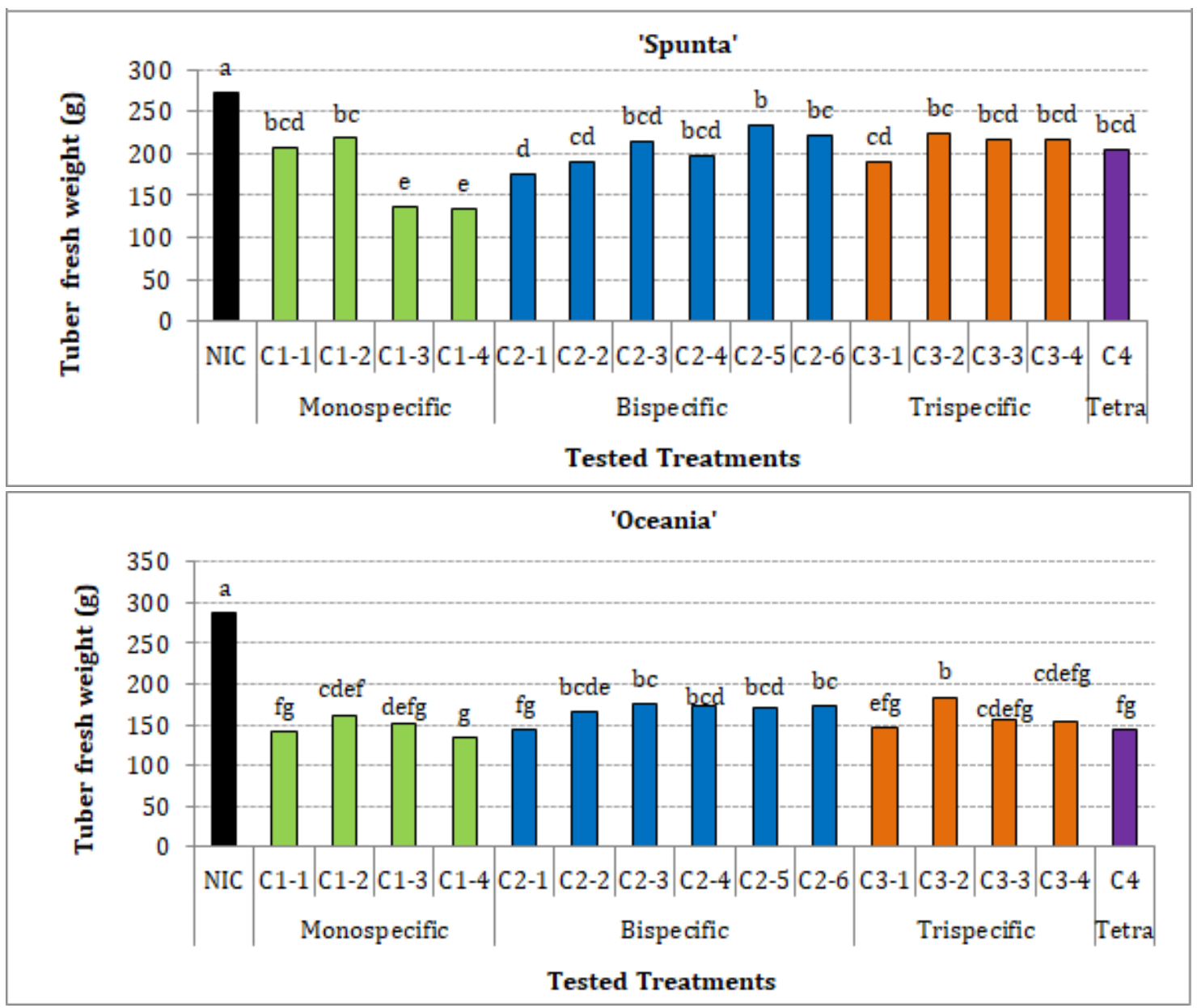

Figure 8. Effect of single and mixed inoculations by four Fusarium species on tuber fresh weight noted on two potato cultivars 75 days post-planting

Bars sharing the same letter are not significantly different according to Duncan's Multiple Range Test at $P \leq 0.05$.

NIC: Uninoculated control, Tetra: Tetraspecific, The composition of all tested inoculation treatments is detailed in Table 1.

Table 3. Behavior of tested potato cultivars to single and mixed infections by Fusarium species determined based on necrosis severity.

\begin{tabular}{|c|c|c|}
\hline Fungal treatment/cultivar & cv. Spunta & cv. Oceania \\
\hline \multicolumn{3}{|l|}{ Monospecific Inoculum } \\
\hline C1-1: F. sol & MS & MS \\
\hline C1-2: F. sam & MS & $\mathrm{T}$ \\
\hline C1-3: F.gra & $\mathrm{T}$ & MS \\
\hline C1-4: F. oxy & $\mathrm{S}$ & MS \\
\hline \multicolumn{3}{|l|}{ Bispecific Inoculum } \\
\hline C2-1: F. sam+F. sol & & $\mathrm{S}$ \\
\hline C2-2: F. sol+F. oxy & S & S \\
\hline C2-3: F. sol+F. gra & S & MS \\
\hline C2-4: F. sam+F. oxy & S & MS \\
\hline C2-5: F. oxy+F. gra & S & MS \\
\hline C2-6: F. sam+F. gra & S & MS \\
\hline \multicolumn{3}{|l|}{ Trispecific Inoculum } \\
\hline C3-1: F. sam +F. sol+F. oxy & $S$ & $M S$ \\
\hline C3-2: F. sol+F. oxy+F.gra & MS & $\mathrm{T}$ \\
\hline
\end{tabular}




\begin{tabular}{llc}
\hline C3-3: . sam+F. oxy+F. gra & S & S \\
C3-4: F. sam+F. sol+F. gra & S & MS \\
Tetraspecific Inoculum & & \\
C4: F. sam+F. sol+F. oxy+F. gra & S & MS \\
\hline
\end{tabular}

T: Tolerant: necrosis index $\geq 2$; MS: Moderately sensitive: $1<$ necrosis index $<2$; $\mathbf{S}$ : Sensitive: necrosis index $\leq 1$. F. sol: F. Solani; F. gra: F. graminearum; F. sam: F. sambuinum; F. oxy: F. oxysporum.

\section{DISCUSSION}

In the last decades, plant pathologists have become increasingly interested in the concept of ecological niche and species coexistence (Kennedy, 2010). In fact, within the same ecological environment, different mechanisms can allocate benefits to one species over another. Indeed, in the current investigation, we studied the interaction that can occur between different Fusarium species colonizing the same ecological niche where a specific interaction leads to the predominance of some species over others. Although it is clear from previous research that individual infection by $F$. oxysporum or $F$. solani can result in wilt chlorosis and premature senescence, effects of $F$. sambucinum and $F$. graminearum, as causative agents of potato Fusarium wilt, was not deeply investigated. In addition, in order to search for tolerant plant material, these most prevalent Fusarium species in Tunisia should be considered in combination as a complex for getting a more sustainable response of potato cultivars to Fusarium wilt. For these reasons, a set of eleven combinations of Fusarium species was studied for the first time in the current investigation.

The results of this study revealed that plant inoculation with various mixtures of Fusariums species led to varied infection severity and cultivar responses. These findings described the involvement of an aggressive association which is the tripartite association composed of $F$. oxysporum, F. sambucinum and F. graminearum. It is important to note that, according to our previous investigations (Mejdoub-Trabelsi and Ben Abdallah, 2016), the mixed infections ranked was being the most aggressive on tubers (as dry rot agents) are different from those classified as aggressive on plants (as wilt agents) in the current investigation. Therefore, in seeking this multi-specific resistance, each Fusarium species should be considered separately. Moreover, presence of $F$. oxysporum, as a component in this association, is not strange because this pathogen has been a fixed component in all mixtures ranked as the most aggressive on plants. Focusing on the type of interactions, the association of $F$. sambucinum, $F$. graminearum and $F$. oxysporum showed increased aggressiveness on plants; suggesting the existence of a synergism between them.

In an attempt to explain this phenomenon, let us remember that on plants, soil factor and the ascending evolution of the species could not be neglected. In fact, $F$. oxysporum, F. sambucinum and F. graminearum do not have the same requirements in the soil and then in the roots and vessels of the plant (Saremi and Amiri, 2010); this could explain their aggressiveness when they have been associated and their needs do not interfere with each other.

These observations lead us to focus on a positive association rather than a negative combination. In this context, Stachowicz (2001) as well as Bruno et al. (2003) suggested that the development of one species may make the environment more favorable for others; which positively affects the structure of the fungal community and increases the aggressiveness of the complex. Since reacting together on plants, competition is by far the relationship that lies between them on plants. Xu et al. (2007) by evaluating the effect of $F$. avenaceum, $F$. culmorum, F. graminearum, and F. poae as simple and mixed inoculations on mycotoxin concentrations, demonstrated that the observed interactions suggest rather the presence of competitive interactions. They recorded a reduction in fungal biomass and a more than 1000 -fold increase in mycotoxin production was detected following co-inoculations.

The interaction of the four Fusarium species differs from those observed on tubers (Mejdoub Trabelsi et al., 2015) where the mixed infection, attributed to $F$. sambucinum and $F$. solani, was found to be the most aggressive on tubers incubated at 20 and $30^{\circ} \mathrm{C}$. Thus, F. sambucinum seems to be the point of intersection of the two most aggressive associations among all tested inocula. This species seems to colonize the tubers perfectly and probably colonize extensively the other plant tissues. Wicklow and Caroll (1981) have long reported that, in competition between fungi, theoretically occupying the same econiche, two features are often intertwined or overlapping i.e. competitive exploitation or interference competition. The first refers to the ability of a given 
species to consume nutrients before another present while the latter involves chemical or adaptive behavior that limits the access of competing species to resources. In addition, the presence of $F$. sambucinum or $F$. oxysporum in the most aggressive complexes does not support the hypothesis that one of them carries the competitive advantage more than the other. The idea of chemical association between these four species cannot be rejected either. This complicity between species can only be affected by factors other than types of associations such as cultivar sensitivity.

Despite the importance of these results, the use of "consortium" of Fusarium species to select the most resistant genotypes to multiple species is more or less questionable according to some authors. Indeed, Reid et al. (1999) indicated that the occurrence of interspecific competition is possible and furthermore, it varies with environmental conditions, including temperature. They suggested the avoidance of complicated interactions that may occur between different species and that the use of species mixtures is not appropriate for the selection of $F$. graminearum and $F$. moniliforme resistant maize. In the current study, after detailing the different effects of single and mixed infections on disease severity and plant growth and production, we suggest variable responses of the tested consortia depending on grown cultivars and growth conditions. In fact, this type of investigations involves several factors that may complicate the interpretation of the obtained results but in this work the Fusarium species complex was monitored taking into account all the factors of the disease triangle. However, these conclusions may be more accurate by performing re-isolations from infected plants to determine which Fusarium species predominates in the complex and may be the more involved in the observed and evaluated symptoms. This complement study may be useful to determine the nature of the relation occurring between tested Fusarium species, positive or negative, in the light of suggestions (Xu et al., 2007).

The current study demonstrated for the first time in the world the specific effects of various single and mixed infections with Fusarium species on wilt severity, subsequent yield loss, and cultivar response. We conclude that Fusarium wilt of potato is expected to be more severe when more than one Fusarium species is present. The relative predominance of $F$. sambucinum may reflect its competitive potential in mixture and its significant involvement in potato Fusarium wilt severity.
Based on the findings of this research and considering the higher positive effects of co-inoculation than individual pathogens, management of the Fusarium wilt should be carried out using more cultivars where more than one species are present. In order to improve potato production in this region, this investigation may be completed by a concomitant monitoring of the evolution of tested inocula in the soil following these recorded interactions.

\section{ACKNOWLEDGMENTS}

This work was funded by the Ministry of Higher Education and Scientific Research of Tunisia through the funding allocated to the research unit UR13AGR09Integrated Horticultural Production in the Tunisian Centre-East, Regional Research Centre on Horticulture and Organic Agriculture of Chott-Mariem, Tunisia.

\section{REFERENCES}

Ayed, F., M. Daami-Remadi, H. Jabnoun-Khiareddine and M. E. Mahjoub. 2006. Effect of potato cultivars on incidence of Fusarium oxysporum f.sp. tuberosi and its transmission to progeny tubers. Journal of Agronomy, 5: 430-34.

Bruno, J. F., J. J. Stachowicz and M. D. Bertness. 2003. Inclusion of facilitation into ecological theory. Trends in Ecology and Evolution, 18: 119-25.

Daami-Remadi, M., F. Ayed, H. Jabnoun-Khiareddine, K. Hibar and M. El Mahjoub. 2006. Comparative susceptibility of some local potato cultivars to four Fusarium species causing tuber dry rot in Tunisia. Journal of Plant Sciences, 1: 306-14.

Daami-Remadi, M. and M. El Mahjoub. 2004. Emergence en Tunisie de Fusarium oxysporum f.sp. tuberosi agent de fletrissure vasculaire des plants et de pourriture seche des tubercules de pomme de terre. EPPO Bulletin, 34: 407-11.

Daami-Remadi, M., S. Zammouri and M. El Mahjoub. 2008. Effect of the level of seed tuber infection by Rhizoctonia solani at planting on potato growth and disease severity. The African Journal of Plant Sciences and Biotechnology, 2: 34-38.

Dung, J. K. S., D. A. Johnson and B. K. Schroeder. 2013. Role of co-infection by Pectobacterium and Verticillium dahliaein the development of early dying and aerial stem rot of Russet Burbank potato. Plant Pathology, 63: 299-307.

Edel-Hermann, V. and C. Lecomte. 2019. Current status 
of Fusarium oxysporum formae speciales and races. Phytopathology, 109: 512-30.

Enya, J., H. Shinohara, S. Yoshida, T. Tsukiboshi, H. Negishi, K. Suyama and S. Tsushima. 2007. Culturable leaf-associated bacteria on tomato plants and their potential as biological control agents. Microbial Ecology, 53: 524-36.

Esfahani, M. N. 2005. Susceptibility assessment of potato cultivars to Fusarium dry rot species. Potato Research, 48: 215-26.

Fisher, M. C., D. A. Henk, C. J. Briggs, J. S. Brownstein, L. C. Madoff, S. L. McCraw and S. J. Gurr. 2012. Emerging fungal threats to animal, plant and ecosystem health. nature, 484: 186-94.

Hou, Q., W. Wang, Y. Yang, J. Hu, C. Bian, L. Jin, G. Li and X. Xiong. 2020. Rhizosphere microbial diversity and community dynamics during potato cultivation. European Journal of Soil Biology, 98: 103176.

Kennedy, P. 2010. Ectomycorrhizal fungi and interspecific competition: Species interactions, community structure, coexistence mechanisms, and future research directions. New Phytologist, 187: 895-910.

Kerkeni, A., M. Daami-Remadi and M. B. Khedher. 2013. In vivo evaluation of compost extracts for the control of the potato fusarium wilt caused by Fusarium oxysporum f. sp. tuberosi. African Journal of Plant Sciences and Biotechnology, 7: 36-41.

Mejdoub-Trabelsi, B. and R. A. Ben Abdallah. 2016. Assessment of the antifungal activity of nonpathogenic potato associated fungi toward Fusarium species causing tuber dry rot disease. Journal of Plant Pathology and Microbiology, 07: 343-49.

Mejdoub-Trabelsi, B., H. Jabnoun-Khiareddine and M. Daami-Remadi. 2012. Effect of Fusarium species and temperature of storage on the susceptibility ranking of potato cultivars to tuber dry rot Potato Pathology. Pest Technology, 6: 41-46.

Mejdoub Trabelsi, B., H. J. Khiareddine and M. D. Remadi. 2015. Interactions between four Fusarium species in potato tubers and consequences for fungal development and susceptibility assessment of five potato cultivars under different storage temperature. Journal of Plant Pathology and Microbiology, 06: 293-97.

Munkvold, G. P. 2016. Fusarium Species and Their Associated Mycotoxins Methods in Molecular
Biology. Springer. New York, USA. pp. 51-106.

Ommati, F. and K. Sharifi. 2008. Determination of species and dispersal of potato Fusarium wilt in Semnan province Proceedings of the 18th Iranian plant protection congress. Hamadan, Iran. pp. 70.

Ommati, F., M. Zaker and A. Mohammadi. 2013. Biological control of Fusarium wilt of potato (Fusarium oxysporum f. sp. tuberosi) by Trichoderma isolates under field condition and their effect on yield. Journal of Crop Protection, 2: 435-42.

Pernezny, K., M. Elliott and A. H. Palmateer, N. 2014. Guidelines for identification and management of plant disease problems: part II. Diagnosing plant diseases caused by fungi, bacteria and virusesUniversity of Florida, Department of Plant Pathology. Florida, USA.

Rahimian, M. K. 1984. Relationship of Verticillium dahliae and Erwinia carotovora pv. carotovora in the early dying disease of potato. Phytopathology, 74: 327-34.

Rampersad, S. 2020. Pathogenomics and management of fusarium diseases in plants. Pathogens, 9: 340-48.

Reid, L. M., R. W. Nicol, T. Ouellet, M. Savard, J. D. Miller, J. C. Young, D. W. Stewart and A. W. Schaafsma. 1999. Interaction of Fusarium graminearum and $F$. moniliforme in maize ears: Disease progress, fungal biomass, and mycotoxin accumulation. Phytopathology, 89: 1028-37.

Rowe, R. C. 1987. Potato early dying: Causal agents and management strategies. Plant disease, 71: 482-92.

Saremi, H. and M. E. Amiri. 2010. Exploration of potato cultivar resistant to the major fungal pathogen on potato wilting disease in Iran. Journal of Food, Agriculture and Environmental, 8: 821-26.

Stachowicz, J. J. 2001. Mutualism, facilitation, and the structure of ecological communities. BioScience, 51: 235-46.

Wicklow, D. and G. C. Caroll. 1981. The fungal community: Its organization and role in the ecosystem.: 855.

Xu, X. M., W. Monger, A. Ritieni and P. Nicholson. 2007. Effect of temperature and duration of wetness during initial infection periods on disease development, fungal biomass and mycotoxin concentrations on wheat inoculated with single, or combinations of, Fusarium species. Plant Pathology, 56: 943-56. 
Xue, R. F., J. Wu, L. F. Wang, M. W. Blair, X. M. Wang, W. De Ge, Z. D. Zhu and S. M. Wang. 2013. Salicylic acid enhances resistance to Fusarium oxysporum f. sp. phaseoli in common beans (Phaseolus vulgaris L.). Journal of Plant Growth Regulation, 33: 470-76.

\section{CONFLICT OF INTEREST}

All authors declare that they have no conflict of interest.

\section{AUTHORS CONTRIBUTIONS}

All authors participated in the design of the study, data collection and data analysis. They read and approved the final manuscript.

Publisher's note: EScience Press remains neutral with regard to jurisdictional claims in published maps and institutional affiliations.

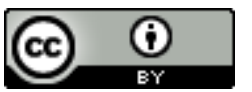

Open Access This article is licensed under a Creative Commons Attribution 4.0 International License, which permits use, sharing, adaptation, distribution and reproduction in any medium or format, as long as you give appropriate credit to the original author(s) and the source, provide a link to the Creative Commons license and indicate if changes were made. The images or other third-party material in this article are included in the article's Creative Commons license, unless indicated otherwise in a credit line to the material. If material is not included in the article's Creative Commons license and your intended use is not permitted by statutory regulation or exceeds the permitted use, you will need to obtain permission directly from the copyright holder. To view a copy of this license, visit http://creativecommons.org/licenses/by/4.0/. 